\title{
A Study of a Fractional-order Model of Coronavius Disease 2019(COVID-19) after Mass Caccination
}

Chunhua Wang ( $\square$ wch1227164@hnu.edu.cn )

Hunan University https://orcid.org/0000-0001-6522-9795

\section{Xiaojuan Ma}

Hunan University

\section{Research Article}

Keywords: Vaccine, COVID-19, Fractional Order, Asymptomatic Patient

Posted Date: March 7th, 2022

DOI: https://doi.org/10.21203/rs.3.rs-1414520/v1

License: (9) This work is licensed under a Creative Commons Attribution 4.0 International License. Read Full License 


\title{
A Study of a Fractional-order Model of Coronavius Disease 2019(COVID-19) after Mass Caccination
}

\author{
Xiaojuan Ma • Chunhua Wang
}

Received: date / Accepted: date

\begin{abstract}
New coronavirus (COVID-19) broke out at the end of 2019. Today, the epidemic has spread all over the world. The number of confirmed cases is 250 million and the number of deaths has reached about 5000000 , which undoubtedly caused serious public health disasters all over the world. In the face of the problem that the virus continues to mutate and the virus spreads faster and survives for a longer time after mutation, as well as the situation of asymptomatic infected persons, the study of the transmission law and epidemic trend of COVID-19 provides theoretical support for how to effectively prevent epidemics, which is of great research value.
\end{abstract}

Most of the previous studies on COVID-19 adopt SIR model and variants of SIR model, and there are few theoretical studies on the impact of asymptomatic infection on the transmission, prevention and control of infectious diseases.Because the time of large-scale vaccination of COVID-19 vaccine in China is about the middle of 2021, so far, no scholars have studied the transmission law and prevention and control of COVID-19 virus after large-scale vaccination. In addition,most researchers use the integer-order models.Generally speaking, integer-order may not capture satisfactory model attributes. Based on this, this paper proposes a SEQIR model with quarantine item, sets up seven fractional order equations, studies and compares the epidemic prevention measures taken by the two places in the face

X. Ma. C. Wang (四) (D.

College of Computer Science and Electronic Engineering $\mathrm{Hu}-$ nan University, Changsha 410082, China

X. Ma

E-mail: mxj1999@hnu.edu.cn

C. Wang

E-mail: wch1227164@hnu.edu.cn of the re outbreak of the epidemic before mass vaccination (Beijing) and after vaccination (Hunan), calculates the size of the basic regeneration number $R_{0}$ in the two places and reports according to the real data of the two places, different numerical simulations are carried out.Studies have shown that mass vaccination and nucleic acid detection can effectively reduce the transmission of the virus. In addition, in the area where the epidemic situation is concentrated, a large number of nucleic acid tests in time can enable asymptomatic infected persons to be detected in time. After isolating asymptomatic infected people, asymptomatic infected people will infect fewer susceptible people.

Keywords Vaccine - COVID-19 - Fractional Order . Asymptomatic Patient

\section{Introduction}

Since the outbreak of novel coronavirus(COVID-19)at the end of 2019, it has aroused widespread concern all over the world.With the increase of close communication between countries and regions and the shortening of distance (convenient transportation), the number and speed of floating population are greatly increased,which leads to the acceleration of cross-regional spread of infectious diseases.Infectious diseases occurring anywhere in the world can spread to other areas in a short time.The existence of asymptomatic infected persons aggravates the spread of infectious diseases, which brings great difficulties to the prevention and control of infectious diseases.For example, the SARS outbreak in 2003 eventually spread to 29 countries in five continents, lasting for about 8 months, with more than 8000 infected people and 774 deaths [1-3].Nowadays, 
COVID-19 is a pandemic all over the world.The practice of epidemic prevention and control has proved that asymptomatic infection plays an important role in the transmission of COVID-19.China screened asymptomatic infected people from the population through large-scale nucleic acid testing, gave the same detailed information as confirmed cases in the epidemic report, tracked their close contacts, isolated them and made medical observation, thus quickly and effectively controlling the epidemic in COVID-19 [4-6].More and more evidence show that asymptomatic infection plays an important role in the spread of infectious diseases. On the one hand, asymptomatic infected persons may be the source of infection.On the other hand, asymptomatic infection can stimulate the body's immune response, improve the group's immune level and control the epidemic of diseases [7-13], but there is little theoretical research on the influence of asymptomatic infection on the spread, prevention and control of infectious diseases.If there are asymptomatic infected people in infectious diseases,its pathogenesis,transmission rule and development trend will be more complicated.Because it is impossible to carry out infectious disease experiments directly in the population, it is particularly important to use the model to study the transmission law of this kind of infectious diseases through theoretical analysis and computer simulation.

Therefore, since the outbreak of COVID-19, many scholars have studied the transmission law and epidemic trend of this kind of virus.For example, Wu et al [14] used the classical SIR model to simulate the pathogenicity and transmission curve of COVID-19, calculate the basic regeneration number, and speculate the infection intensity, peak time, epidemic end time,etc; Leung et al. [15] Based on SIR model, considering that the disease has incubation period, added the patients with incubation period to establish SEIR model, used the case and travel data in this period, and estimated the basic regeneration number by Markov chain Monte Carlo method;Liu et al. [16] used Bayesian SEIR epidemic dynamics model to reconstruct the local transmission trend of COVID-19 in nine regions of the UK. The results showed that compared with China, the UK has a relatively high basic regeneration number. With the implementation of mandatory blockade and isolation control measures, the basic regeneration number will be reduced, and can effectively curb the spread of the epidemic; In literature [17,18], domestic researchers proposed a dynamic model of infectious diseases with time lag term, analyzed the effectiveness of epidemic prevention and control intervention, and inferred the epidemic development trend of countries with different intervention means. The results show that early detec- tion, early isolation and strengthening prevention and control have played a very important role in blocking the spread of the epidemic situation.Jaouad Danane et al. Used the SEIR model of fractional derivative to consider the impact of government action characterized by the term $(1-\rho)(1-D / N)^{k}$ on infectious diseases, and verified that government action and population response quality may play an important role in reducing the severity of infection [19,20];Dr. Li Feng [21] established COVID-19 infectious disease dynamic model for asymptomatic infected people by using SIR model with integer derivative.Studies have shown that large-scale nucleic acid detection and isolation of a large number of susceptible people play a very important role in the prevention and control of epidemic.However, these measures require a lot of manpower, material resources and financial resources. In addition, the application of fractional order in dynamics also has a good effects. Yu et al. [22-24] showed that electromagnetic radiation can not only change the number of equilibrium points in neural network, but also affect the dynamic behavior of neural network. Lin et al. [25-27] discussed in detail the latest progress of neuron and neural network models on neurodynamics, which is helpful to apply dynamics to the study of brain function.Xie et al. [28]proposed a fractional order multistable multi stable local active memristor is proposed.The experimental results show that, compared with the integer memristors, the fractional memristors have stronger local activity and greater nonvolatile storage characteristics.

However, most of the previous studies on COVID19 adopt the SIR model and variants of the SIR model, and there are few theoretical studies on the impact of asymptomatic infection on the transmission and prevention and control of infectious diseases. As the largescale vaccination of COVID-19 vaccine is in the middle and early stage of 2021, so far, no scholars have studied the transmission law and prevention and control of COVID-19 virus after large-scale vaccination. In addition, most researchers use integer-order models. Generally speaking, integer order may not capture satisfactory model attributes. Based on this, this paper puts forward a SEQIR model with isolation term uses fractional order to model, establishes seven fractional order equations, and studies and compares the epidemic prevention measures taken by the two places in the outbreak again (Beijing) and after mass vaccination (Hunan). Firstly, the expression of the basic regeneration number $R_{0}$ is derived by using the next generation matrix method, and then it is proved that it is locally asymptotically stable when $R_{0}<1$. Then, the size of the basic regeneration number $R_{0}$ in the two places is calculated and the sensitivity of its parameters 
is analyzed. Then, according to the real data reports of the two places, different numerical simulations are carried out. Finally, the effectiveness of epidemic prevention measures was evaluated through the experimental comparison.

\section{COVID-19 model of memory dependence}

In this paper, the factors of vaccination are considered, and the SEQIR model with isolation term is established for COVID-19, as shown in the figure below:

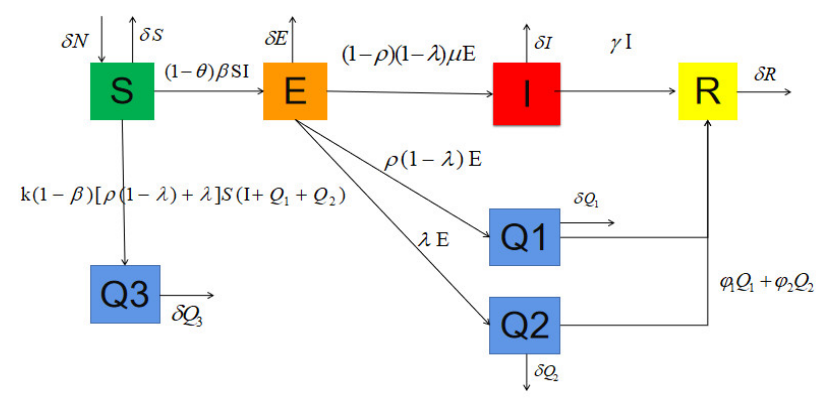

Fig. 1 Transfer diagram between COVID-19 compartments

In the picture above, $\mathrm{S}$ represents susceptible individuals, E represents exposed individuals, and Q represents isolated individuals (where Q3 represents isolated susceptible individuals, Q1 represents isolated symptomatic infected persons and Q2 represents isolated asym tomatic infected persons), I represents infected individuals and $\mathrm{R}$ represents removed individuals. Fractional model is considered to study the abnormal behaviors of mathematical tools observed in many physical processes with remarkable memory and genetic characteristics. Therefore, according to the time propagation map of each chamber, the following seven fractional equations are listed.

$$
\left\{\begin{array}{l}
D^{\alpha} S=-(1-\theta) \beta(t) \frac{S}{N} I-K_{q} \frac{S}{N} I+\delta N-\delta S \\
D^{\alpha} E=(1-\theta) \beta(t) \frac{S}{N} I-\alpha_{1} \mu E-\alpha_{2} E-\lambda E-\delta E \\
D^{\alpha} I=(1-\rho)(1-\lambda) \mu E-\gamma I-\delta I \\
D^{\alpha} R=\gamma I+\phi_{1} Q_{1}+\phi_{2} Q_{2}-\delta R \\
D^{\alpha} Q_{1}=\rho(1-\lambda) E-\phi_{1} Q_{1}-\delta Q_{1} \\
D^{\alpha} Q_{2}=\lambda E-\phi_{2} Q_{2}-\delta Q_{2} \\
D^{\alpha} Q_{3}=k[(1-\beta) \rho(1-\lambda)+\lambda] \frac{S}{N} I-\delta Q_{3}
\end{array}\right.
$$

In the formula, $\sigma$ represents the birth rate and death rate (assuming that the birth rate is equal to the death rate), $\theta$ represents the vaccination rate, $\beta$ Represents the infection coefficient, $\mu$ represents the latent coefficient, $\lambda$ represents the detection rate of uninfected people, $\gamma$ represents the self-healing rate, $\phi_{1}$ represents the cure rate of asymptomatic infected people, $\phi_{2}$ represents the recovery rate of symptomatic infected people, $\rho$ represents the daily accounting and detection rate, $K$ represents the isolation coefficient, and $N$ represents the total number. Let : $\alpha_{1}=(1-\rho)(1-\lambda), \alpha_{2}=\rho(1-\lambda)+$ $\lambda, \alpha_{3}=\rho(1-\lambda), K_{q}=k(1-\beta) \alpha_{2}$.
The equation obeys a nonnegative initial condition, operator $\mathrm{D}^{\alpha}$ express $\alpha$ Caputo fractional Order:

$$
D_{t}^{\alpha} f(t)=\frac{1}{\Gamma(1-\alpha))} \int_{0}^{t} t-s^{-\alpha} f(s) d s \quad \alpha \subseteq(0,1)
$$

\section{Basic regeneration number and equilibrium stability}

\subsection{Basic regeneration number}

The basic reproduction number $R_{0}$ represents the average number of patients infected during the illness, so $R_{0}=1$ can be used as the threshold of disease death. When $R_{0}<1$ loccurs, the disease gradually spreads, and eventually becomes endemic. When $R_{0}<1$ occurs, the illness gradually disappears naturally. Therefore, it is very necessary to estimate the basic reproduction number $R_{0}$. Since the birth rate and mortality rate have little influence on this model, we use the next generation matrix $F V^{-1}$ to evaluate the basic reproduction number $R_{0}$ [29].The formula is as follows: $R_{0}=\rho F V^{-1}$.

$$
F=\left(\begin{array}{cc}
0 & (1-\theta) \beta \\
0 & 0
\end{array}\right) V=\left(\begin{array}{cc}
\alpha_{1} \mu+\alpha_{2} & 0 \\
-\alpha_{1} \mu & 0
\end{array}\right)
$$

According to the method of next generation matrix proposed in reference [29-31], the expression of basic - regeneration number $R_{0}$ is obtained as follows:

$$
R_{0}=\frac{(1-\theta) \beta \alpha_{1} \mu}{\alpha_{1} \mu \gamma+\alpha_{2} \gamma}
$$

\subsection{Equilibrium stability}

Next, we prove the local asymptotic stability of the disease

Theorem 3.1: [32]A point $u^{*}$ is the equilibrium point of Caputo fractional dynamical system $\mathrm{D}^{\alpha} \mathrm{u}(\mathrm{t})=\mathrm{f}(\mathrm{t}, \mathrm{u})$, if and only if $\mathrm{f}\left(\mathrm{t}, \mathrm{u}^{*}\right)=0$.

According to the theorem, $u^{*}=(1,0,0,0,0,0,0)$ is an equilibrium point of (1). This is called disease-free balance.

Next, the local asymptotic stability of the diseasefree equilibrium of the system is proved: firstly, the equilibrium point of the system is linearized [33] :

$\mathrm{D}^{\alpha} \mathrm{u}(\mathrm{t})=\mathrm{f}(\mathrm{u}), 0<\alpha<1$;

Then apply the theorem of Matignon [34]:

Theorem 3.2: Consider the following fractional-order system :

$\mathrm{D}^{\alpha} \mathrm{u}(\mathrm{t})=\mathrm{f}(\mathrm{u}), 0<\alpha<1 ;$

If all eigenvalues of Jacobian matrix $J u^{*}=\left.\frac{\partial f}{\partial u}\right|_{\mathrm{u}^{*}}$ that all conditions are met: 
$|\arg \lambda|>\alpha \frac{\pi}{2}$

then $u^{*}$ is locally asymptotically stable.

Based on lemma 3.2, the stability results of system (1) are obtained as follows: the disease-free equilibrium $u^{*}=(1,0,0,0,0,0,0)$ of model $(1)$ is locally asymptotically stable when $R_{0}<1$.

Proof : Linearize the model at $u^{*}=(1,0,0,0,0,0,0)$, and obtain the Jacobian matrix at $u^{*}$ :

$J u^{*}=\left(\begin{array}{ccccccc}-\left(\alpha_{1} \mu+\alpha_{2}\right)-\delta & (1-\theta) \beta & 0 & 0 & 0 & 0 & 0 \\ \alpha_{1} \mu & -\gamma-\delta & 0 & 0 & 0 & 0 & 0 \\ 0 & \gamma & -\delta & \phi_{1} & \phi_{2} & 0 & 0 \\ \alpha_{1} & 0 & 0 & -\phi_{1}-\delta & 0 & 0 & 0 \\ \lambda & 0 & 0 & 0 & \phi_{2}-\delta & 0 & 0 \\ 0 & k_{q} & 0 & k_{q} & k_{q} & -\delta & k_{q} \\ 0 & -(1-\theta) \beta-K & 0 & -k_{q} & -k_{q} & 0 & -\delta\end{array}\right)$

Eigenvalues obtained by calculation: $\lambda_{1}=-\left(\alpha_{1} \mu+\right.$ $\left.\alpha_{2}\right)-\delta, \lambda_{2}=-\gamma-\delta, \lambda_{3}=\lambda_{6}=\lambda_{7}=-\delta, \lambda_{4}=$ $-\phi_{1}-\delta, \lambda_{5}=-\phi_{2}-\delta$.

When $R_{0}<1$, all eigenvalues of Jacobian matrix are negative. It can be seen from theorem 3.2 that when $R_{0}<1$, the disease-free equilibrium point of the model is gradually stable.

\section{Model simulation}

4.1 Introduction to the solution method for fractional systems

The numerical algorithm for the fractional differential equations applied in this paper is the prediction-correction method proposed by Diethelm and Freed.The method is a combination of some product integral rules, commonly referred to as the fractional Adams-Bashforth-Moulton approach [35], The basic principles are described as follows:

First, we give a fractional differential equation:

$$
D_{t_{0}}^{\alpha}\left(y-y_{0}\right)(t)=f(t, y(t)), \quad y\left(t_{0}\right)=y_{0}
$$

Using the basic theorem of fractional calculus, the above differential equations can be transformed into:

$\frac{1}{\Gamma(-\alpha)} \int_{t_{0}}^{t} \frac{y(u)-y_{0}}{(t-u)^{\alpha+1}} d u=f(t, y(t)), y\left(t_{0}\right)=y_{0}$

We can also apply a fractional integral operator to the differential equation, incorporating the initial conditions, and thus converting the equation into an equivalent equation:

$y(t)=y\left(t_{0}\right)+\frac{1}{\Gamma(-\alpha)} \int_{t_{0}}^{t}(t-u)^{\alpha-1} f(u, y(u)) d u$

The key to this method is to take the equivalent equation (7) instead of the original fractional differential equation (5), and to implement a product integral method for the latter.This method is simply use the product trapezoid quadrution formula with nodes $t_{j}(\mathrm{j}=0,1, \ldots, \mathrm{n}+1)$,Instead of the integral, according to the weight function $\left(t_{n+1}-\cdot\right)^{\alpha-1}$.

$\int_{t_{0}}^{t_{n+1}}\left(t_{n+1}-u\right)^{\alpha-1} g(u) d u$

$\approx \int_{t_{0}}^{t_{n+1}}\left(t_{n+1}-u\right)^{\alpha-1} g_{n+1}(u) d u$

Further calculation of formula. (8) can be obtained:

$a_{j, n+1}=\int_{t_{0}}^{t_{n+1}}\left(t_{n+1}-u\right)^{\alpha-1} \phi_{j, n+1}(u) d u$

And:

$\phi_{j, n+1}(u)= \begin{cases}\left(u-t_{j-1}\right) /\left(t_{j}-t_{j-1}\right) & \text { if } t_{j-1}<u<t_{j}, \\ \left(t_{j+1}-u\right) /\left(t_{j+1}-t_{j}\right) & \text { if } t_{j}<u<t_{j+1}, \\ 0 & \text { otherwise }\end{cases}$

In the isometric node $t_{j}=t_{0}+j h$ has some fixed $\mathrm{h}$ of the cases:

$a_{j, n+1}=\left\{\begin{array}{lc}\frac{h^{\alpha}}{\alpha(\alpha+1)}\left(n^{\alpha+1}-(n-\alpha)(n+1)^{\alpha}\right) & \text { if } j=0, \\ \frac{h^{\alpha}}{\alpha(\alpha+1)} & \text { if } j=n+1\end{array}\right.$

When $1 \leqslant j \leqslant n$, we have:

$$
\begin{aligned}
a_{j, n+1}= & \frac{h^{\alpha}}{\alpha(\alpha+1)}\left((n-j+2)^{\alpha+1}\right. \\
& \left.-2(n-j+1)^{\alpha+1}+(n-j)^{\alpha+1}\right)
\end{aligned}
$$

We then present our correction formula,namely, fractional variants of the Adams-Morton method:

$$
\begin{aligned}
y_{n+1}= & y_{0}+\frac{1}{\Gamma(\alpha)}\left(\sum_{j=0}^{n} a_{j, n+1} f\left(t_{j}, y_{j}\right)\right. \\
& \left.+a_{n+1, n+1} f\left(t_{n+1}, y_{n+1}^{p}\right)\right)
\end{aligned}
$$

For the prediction formula of the $y_{n+1}^{p}$, Replace the integral to the right of Eq.(7) Rule made by the product rectangles, i. e.

$\int_{t_{0}}^{t_{n+1}}\left(t_{n+1}-u\right)^{\alpha-1} g(u) d u \approx \sum_{j=0}^{n} b_{j, n+1} g\left(t_{j}\right)$

Now: 


$$
\begin{aligned}
b_{j, n+1} & =\int_{t_{j}}^{t_{j+1}}\left(t_{n+1}-u\right)^{\alpha-1} d u \\
& =\frac{1}{\alpha}\left(\left(t_{n+1}-t_{j}\right)^{\alpha}-\left(t_{n+1}-t_{j+1}\right)^{\alpha}\right.
\end{aligned}
$$

After the (22) collation can get a simpler expression namely:

$b_{j, n+1}=\frac{h^{\alpha}}{\alpha}\left((n+1-j)^{\alpha}-(n-j)^{\alpha}\right)$

Thus, up to the predictive formula:

$y_{n+1}^{p}=y_{0}+\frac{1}{\Gamma(\alpha)} \sum_{j=0}^{n} b_{j, n+1} f\left(t_{j}, y_{j}\right)$

This completes the basic description of the algorithmï $\frac{1}{4}$ We must first calculate the predictor from the formula for (17) $y_{n+1}^{p}$. Then we compute $f\left(t_{n+1}, y_{n+1}^{p}\right)$, use it to determine the calibrator by formula for $(13) y_{n+1}$, The final calculation $f\left(t_{n+1}, y_{n+1}\right)$, It is then used in the next integration step. The stability analysis of the method has been analyzed by the author of paper [36], and here We do not do much.

\subsection{Source of the various parameters of the system}

Relevant data of COVID-19 of Beijing and Hunan Province can be obtained from Beijing Health and Health Commission and Hunan Health and Health Commission. Including newly confirmed cases, suspected cases, death cases, cured cases, asymptomatic infected persons, accumulated close contacts and other relevant information.

The total population of Beijing is $21.536 \operatorname{million}(N=$ $\left.2.1536 \times 10^{7}\right)$.From June 11 to July 9, 2020, 335 locally confirmed cases, 52 asymptomatic infections and no deaths were reported in Beijing.According to Beijing's data, the average time from infection to virus detection is 10.8 days $(\lambda=1 / 10.8)$, About 300000 people are tested for nucleic acid every $\operatorname{day}\left(\rho=3 \times 10^{5} / N\right)$.Initial value of Beijing: $\mathrm{R}(0)=0, \mathrm{~S}(0)=2.1536 \times 10^{7}$.

The total population of Hunan is $66.445 \operatorname{million}(N=$ $\left.6.6445 \times 10^{7}\right)$.From July $28^{t h}, 2021$ to August $25^{\text {th }}, 2021$, Hunan Province reported 110 locally confirmed cases and 19 asymptomatic infected cases.According to $\mathrm{Hu}-$ nan's data, the average time from infection to virus detection is 8 days $(\lambda=1 / 8)$, About 960000 people are tested for nucleic acid every day $\left(\rho=9.6 \times 10^{5} / N\right)$. Initial value of Hunan Province: $\mathrm{S}(0)=6.6445 \times 10^{7}, \mathrm{R}(0)=0$. Because the vaccination situation has been reflected in the estimated infection coefficient based on the actual infection data $\beta$. Therefore, in the simulation, the vaccination ratio of the two places was taken as 0 every day.

Table 1 Parameters of Beijing and Hunan models

\begin{tabular}{llll}
\hline Parameter & Beijing & Hunan & Source \\
\hline$\beta$ & 0.0639 & 0.0390 & Estimate(MCMC) \\
$\theta$ & 0 & 0.4357 & News Network \\
$\mu$ & $1 / 7$ & $1 / 7$ & Network query \\
$\lambda$ & $1 / 10.8$ & $1 / 8$ & Estimate(MCMC) \\
$\rho$ & $3 \times 10^{5} / N$ & $9.6 \times 10^{5} / N$ & Health Committee \\
$\gamma$ & $1 / 21$ & $1 / 21$ & Estimate(MCMC) \\
$\phi_{1}$ & $1 / 11$ & $1 / 8$ & Health Committee \\
$\phi_{2}$ & $1 / 11$ & $1 / 8$ & Health Committee \\
\hline
\end{tabular}

4.3 numerical simulation

Using the numerical techniques mentioned above and the values of each parameter obtained, the model studied is solved by Matlab software programming, and the results of this model are calculated. The following is the fitting of the total number of infected individuals with symptoms in Beijing and Hunan based on the fractional system. The fitting results are shown in Fig.2 and Fig.3:

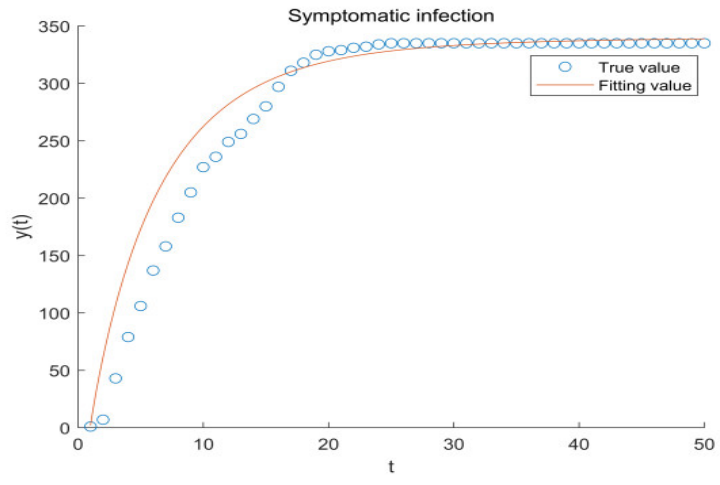

Fig. 2 Fitting results between the total number of symptomatic COVID-19 infected persons in Beijing and the model solution

\section{Sensitivity analysis}

In order to study the influence of model parameters on the system, this paper makes a sensitivity analysis of its parameters $\rho, \beta$ and $\lambda$. The main purpose is to find out which parameters of the model can significantly change the infection kinetics, so as to find out the parameters that have a greater influence on the model.According to 


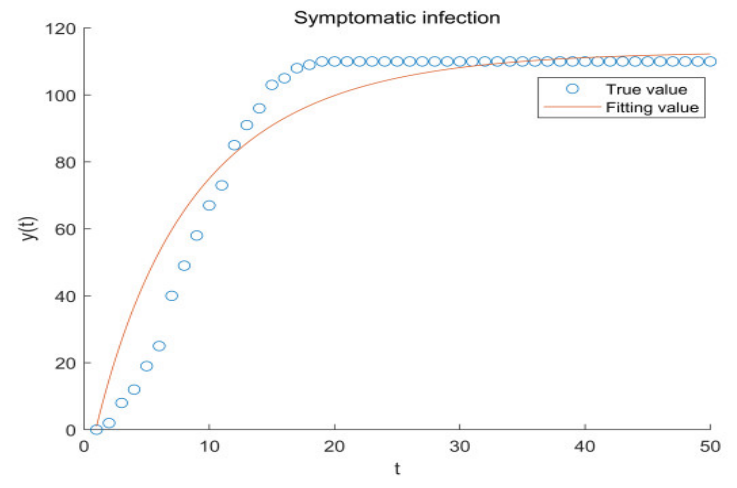

Fig. 3 Fitting results between the total number of symptomatic COVID-19 infected persons in Hunan and the model solution

the calculation formula of normalized sensitivity index:

$\phi_{v}=\frac{\partial R_{0}}{\partial v} \frac{v}{R_{0}}$

We get the following equations:

$\phi_{\beta}=1$

$\phi_{\rho}=-\frac{\rho}{(1-\rho)[(1-\lambda) \mu+\lambda+(1-\mu)(1-\lambda) \rho)]}$

$\phi_{\lambda}=-\frac{\lambda}{(1-\lambda)[[(1-\rho)+\lambda(\rho-1)] \mu+\rho-\lambda(\rho-1))]}$

Table 2 Sensitivity values of $R_{0}$ corresponding parameters in Beijing and Hunan

\begin{tabular}{lll}
\hline Parameter & SensitivityIndex $(\mathrm{B})$ & SensitivityIndex $(\mathrm{H})$ \\
\hline$\beta$ & 1 & 1 \\
$\rho$ & -0.0606 & -0.0757 \\
$\lambda$ & -0.4378 & -0.2459 \\
\hline
\end{tabular}

Table 3 Corresponding values of $R_{0}$ in Beijing and Hunan

\begin{tabular}{ll}
\hline $\mathrm{R}_{0}$ & value \\
\hline Beijing & 0.7371 \\
Hunan & 0.1624 \\
\hline
\end{tabular}

According to the contents in the above table, draw the following pictures:

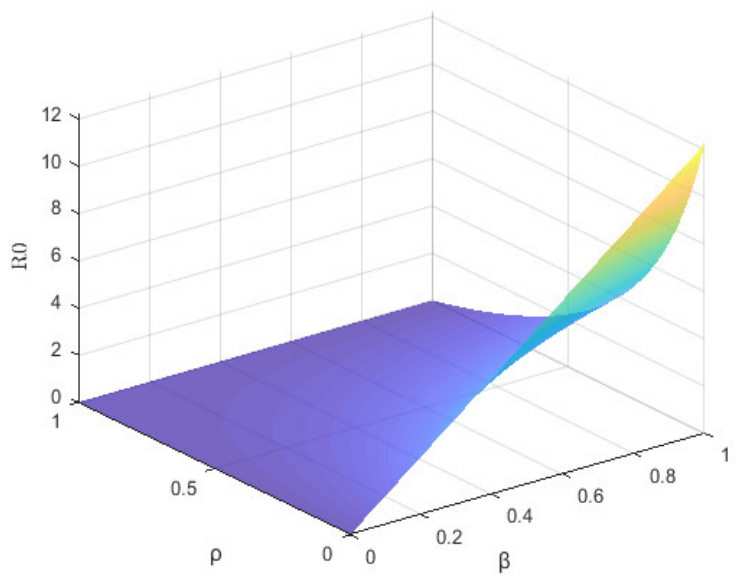

Fig. $4 \quad R_{0}$ about $\beta$ and $\rho$ of sensitivity (Beijing)

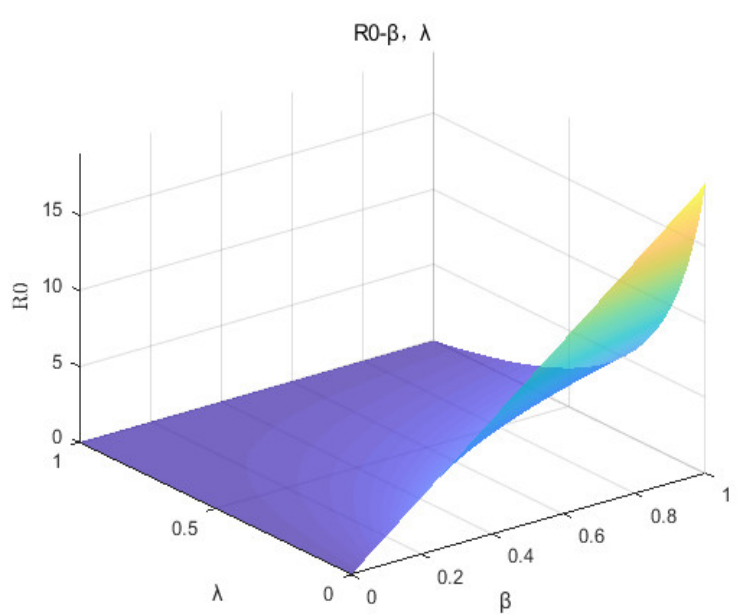

Fig. $5 \quad R_{0}$ about $\beta$ and $\lambda$ of sensitivity (Beijing)

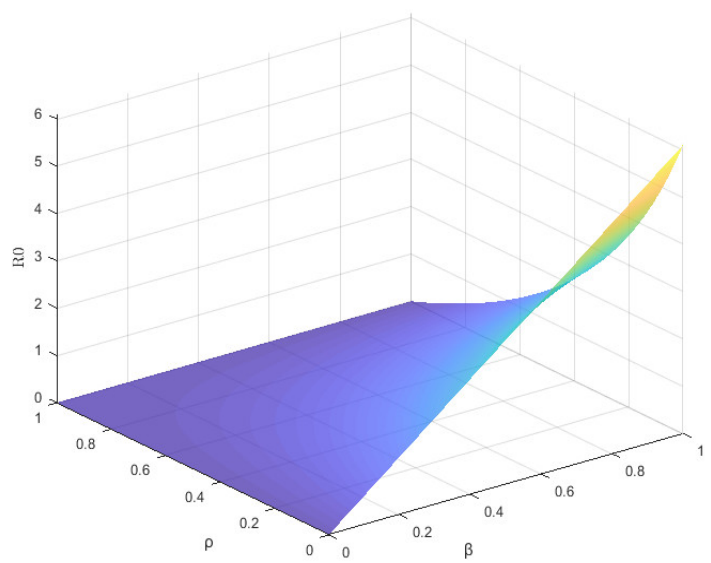

Fig. $6 R_{0}$ about $\beta$ and $\rho$ of sensitivity (Hunan) 


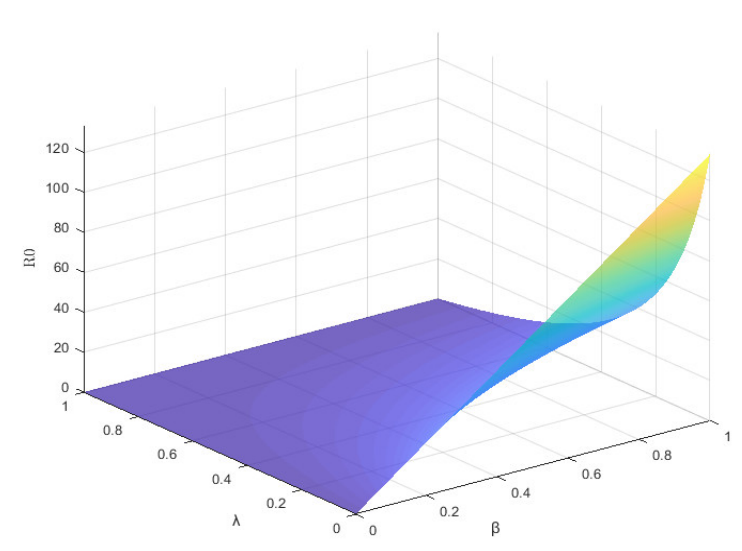

Fig. $7 R_{0}$ about $\beta$ and $\lambda$ of sensitivity (Hunan)

As can be seen from table 2 , the parameter $\beta$ is a positive sensitivity index, and other parameters $\rho$ and $\lambda$ are a negative sensitivity index. It is worth noting that the absolute value range of parameter $\beta$ is large, which means that it is the most sensitive parameter in the model under consideration. On the other hand, increasing the value of parameters $\rho$ and $\lambda$ will reduce $R_{0}$, which will lead to a decrease of infection degree.

The sensitivity analysis of Beijing and Hunan showed From Figure 4 to Figure 7 , respectively $R_{0}$ about $\beta$ and $\rho$ And $R_{0}$ about $\beta$ and $\lambda$.According to the sensitivity chart. It can be noted that for $\beta=1$ and $\rho=0$, the $R_{0}$ âs value can extend to 12 . When $\rho$ from 0 to 1 , we can see that the value of $R_{0}$ gradually decreases and finally tends to 0 .Similarly, if $\beta=1$ and $\lambda=0$, then $R_{0}$ will become very large. As $\lambda$ from 0 to 1 , we can see that the value of $R_{0}$ gradually decreases and finally tends to 0 , which shows that the detection rate of undetected infected persons and the number of nucleic acid detection quantities play a very important role in controlling the epidemic situation.

\section{Evaluate the effectiveness of prevention and control measures}

From Figure 8 to Figure 15, it can be seen that the recovering population of Beijing and Hunan has obviously increased, which means that most of the population will recover. On the other hand, the number of infected and exposed individuals and the population in the incubation period decreased significantly. It shows that the disease will recover gradually, which is consistent with the previous results of basic regeneration number $R_{0}<1$.

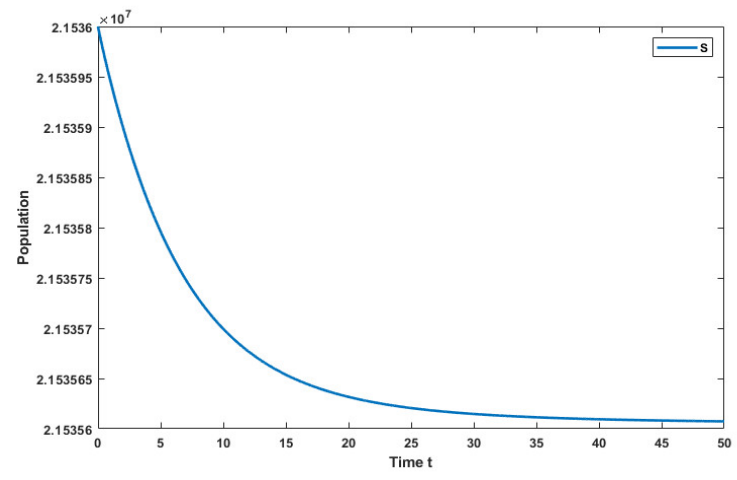

Fig. 8 Beijing $\alpha=0.95$ variation diagram of S compartment with time $\mathrm{t}$

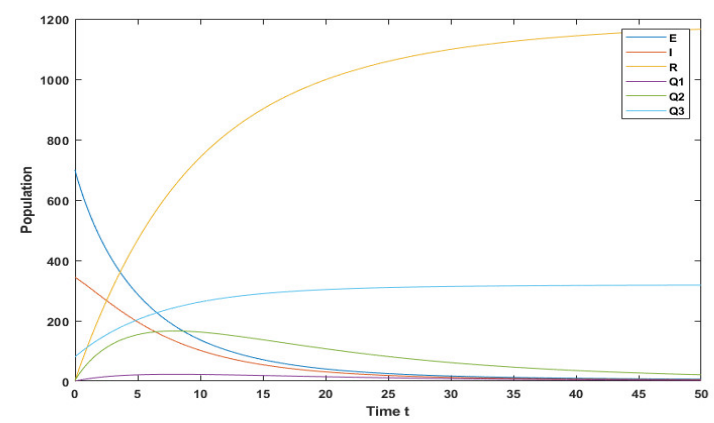

Fig. 9 Beijing $\alpha=0.95$ variation diagram of E,I,R,Q1,Q2,Q3 compartment with time $\mathrm{t}$

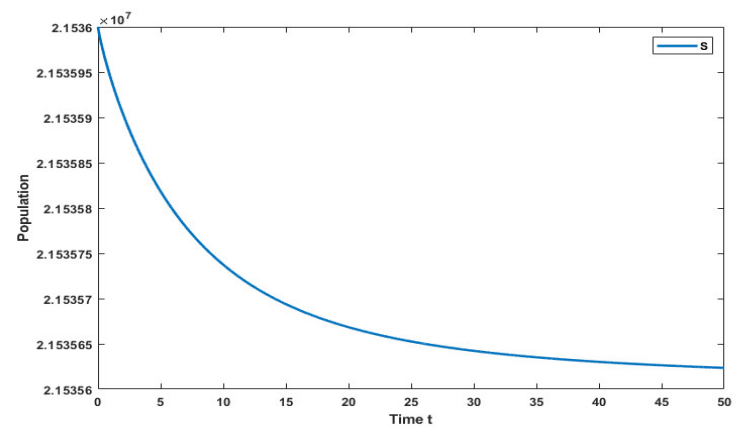

Fig. 10 Beijing $\alpha=0.85$ Variation diagram of S compartment with time

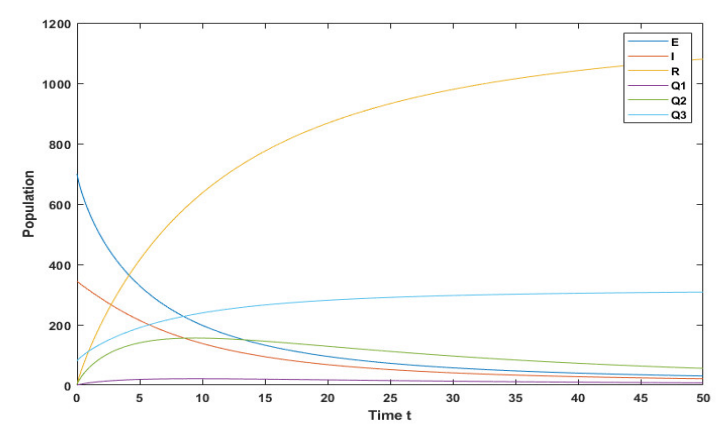

Fig. 11 Beijing $\quad \alpha=0.85$ variation diagram of E,I,R,Q1,Q2,Q3 compartment with time t 


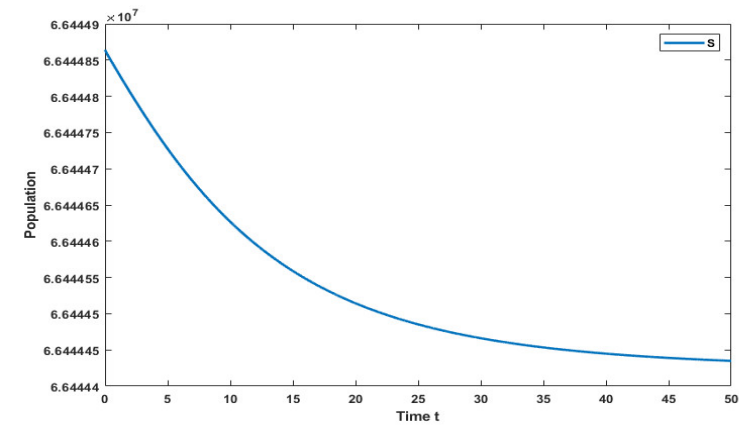

Fig. 12 Hunan $\alpha=0.95$ variation diagram of $\mathrm{S}$ compartment with time $\mathrm{t}$

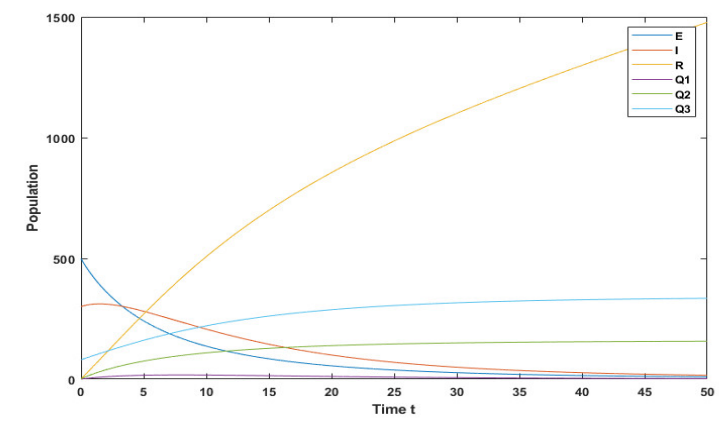

Fig. 13 Hunan $\alpha=0.95$ variation diagram of E,I,R,Q1,Q2,Q3 compartment with time $\mathrm{t}$

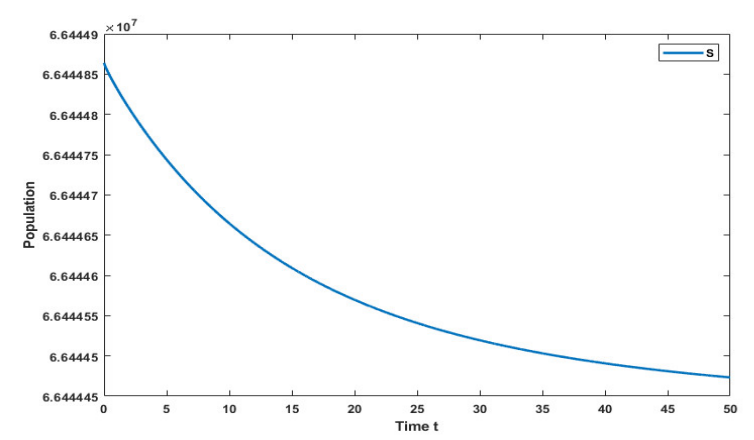

Fig. 14 Hunan $\alpha=0.85$ variation diagram of S compartment with time $t$

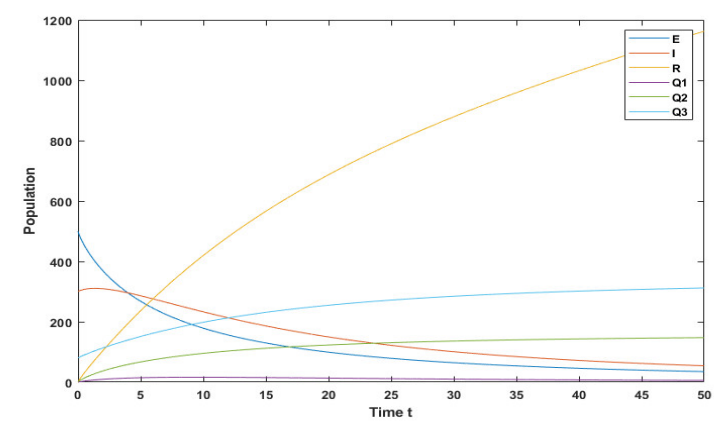

Fig. 15 Hunan $\alpha=0.85$ variation diagram of E,I,R,Q1,Q2, Q3 compartment with time $\mathrm{t}$
In Figure 16 and Figure 17, we show the dynamic behavior of the compartments of the infected population and the recovered population in Hunan when changing the size of $\alpha$. The number of infected people and recovery people will also be reduced.Therefore, the larger the $\alpha$ is, the more healthy individuals there are.

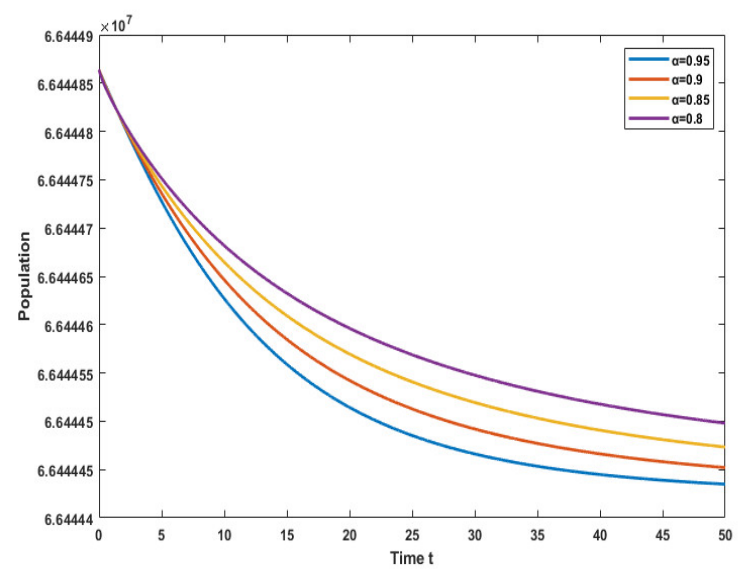

Fig. 16 Different $\alpha$ in Hunan Change trend of S compartment under

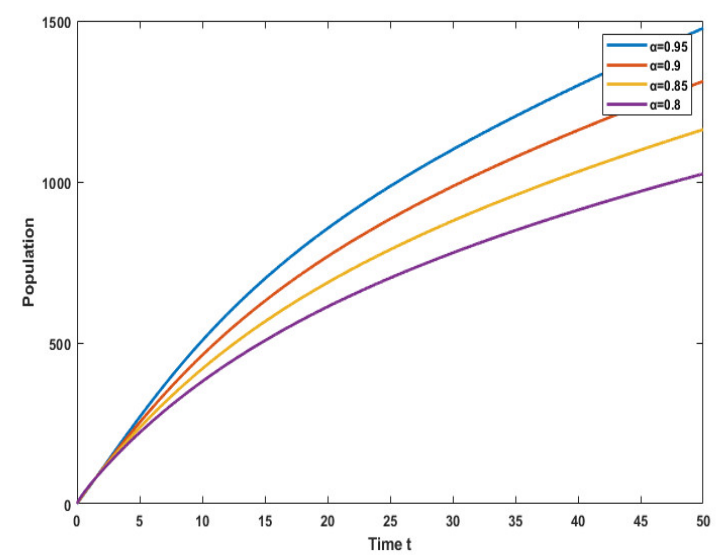

Fig. 17 Different $\alpha$ in Hunan Change trend of $\mathrm{R}$ compartment under

Figure18 to Figure20 illustrate that when COVID19 breaks out, the government takes corresponding measures in time, such as the behavior of susceptible and infected people under different daily nucleic acid detection quantities.It can be seen that if the government takes strong action to carry out a large number of nucleic acid detection for people in medium and high-risk areas, the number of susceptible people will be limited, and asymptomatic infected people will be detected through a large number of accounting and testing. Therefore, after asymptomatic infected people 
are detected, they will be isolated, and accordingly, the number of people infected by asymptomatic infections will be reduced.Thus, the government carries out a large number of nucleic acid tests for people in medium and high risk areas, which is very important for the prevention and control of COVID-19.

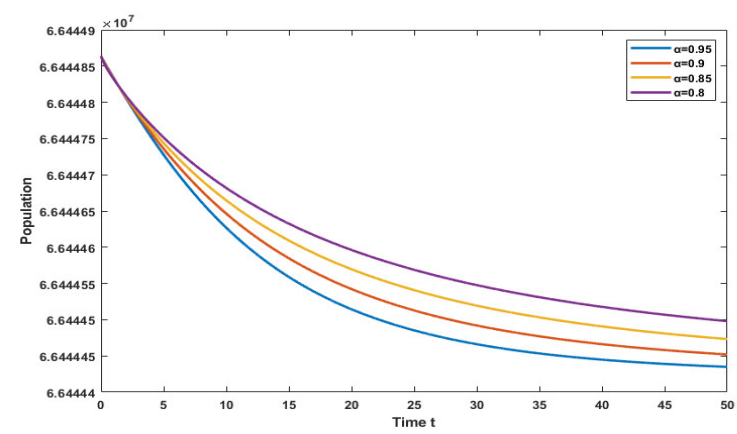

Fig. 18 Different $\rho$ in Hunan Change trend of S compartment

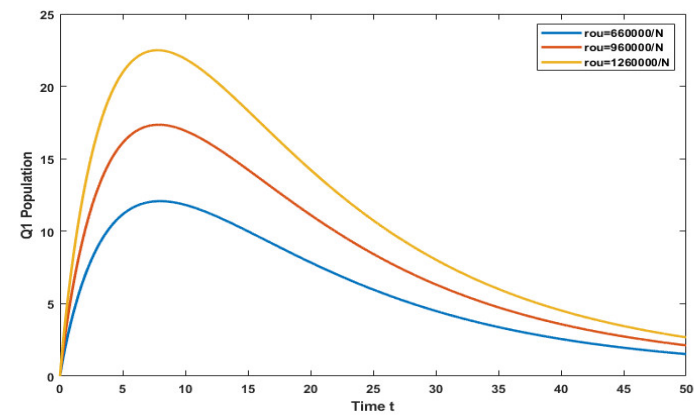

Fig. 19 Different $\rho$ in Hunan Change trend of Q1 compartment under

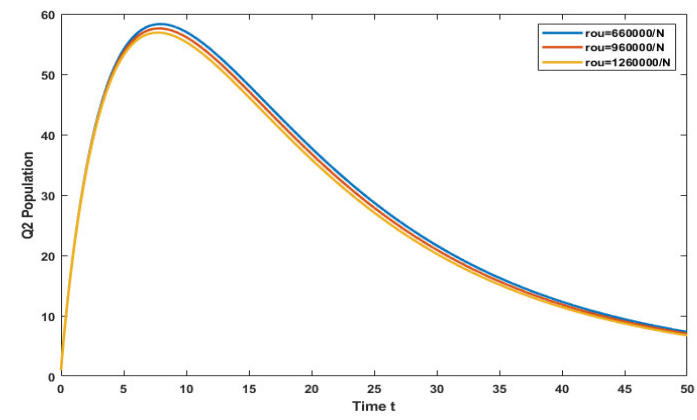

Fig. 20 Different $\rho$ in Hunan Change trend of Q2 compartment under

Because in June 2020, Beijing had not been vaccinated on a large scale, in the face of the outbreak of the epidemic again, the government took strong measures, and the daily accounting and testing reached more than 300,000 people, which made the epidemic under control within 26 days. After the outbreak of the Hunan epidemic in August, 2021, the number of daily nucleic acid tests in Hunan province reached 960,000 people, and the vaccine was vaccinated on a large scale. Although the vaccination proportion in Hunan was about $40 \%$, it also brought strength to the prevention and control of the epidemic.According to the data of the two places estimated by the system, the infection coefficient of Hunan $(\beta=0.0390)$ and basic regeneration number $\left(R_{0}=0.1624\right)$ are smaller than the infection coefficient of Beijing $(\beta=0.0639)$ and the basic regeneration number $\left(R_{0}=0.7371\right)$. The epidemic situation in Hunan was controlled within 18 days, and zero growth was achieved in $19^{t} h$ day. Moreover, the number of infected persons (110) in Hunan is 0.33 times that of infected persons (335) in Beijing, indicating that mass vaccination plays a very important role in epidemic prevention and control.

\section{Conclusion}

In this paper, seven fractional equations are established by using the SEQIR model, and the epidemic prevention measures taken by the two places in the outbreak of the epidemic again (Beijing) and after mass vaccination (Hunan) are studied and compared. Firstly, the expression of the basic regeneration number $R_{0}$ is derived by using the next generation matrix method, and then it is proved that it is locally asymptotically stable when $R_{0}<1$, Then calculate the size of the basic reproduction number $R_{0}$ in the two places, analyze the sensitivity of $R_{0}$ and its parameters $\beta, \rho$ and $\lambda$, and find out which parameter of the model can significantly change the infection dynamics.Finally, the effectiveness of epidemic prevention measures is evaluated through the comparison of experiments.Studies have shown that large-scale vaccination and nucleic acid testing can effectively reduce the spread of the virus. In addition, in the area where the epidemic situation is concentrated, a large number of accounting and testing in time can enable asymptomatic infected persons to be detected in time. After isolating asymptomatic infected persons, asymptomatic infected persons can be reduced to infect more susceptible persons.

\section{Data Availability Statements}

All data generated or analysed during this study are included in this published article (and its supplementary information files). 


\section{Compliance with ethical standards}

Conflict of interest The authors declare that they have no conflicts of interest.

\section{References}

1. Zhong, N.S., Zheng, B.J., Li, Y.M., Poon, L.L.M., Xie, Z.H., Chan, K.H., Li,P. H., Tan, S.Y., Chang, Q., Xie.J.P.:Epidemiology and cause of severe acute respiratory syndrome (SARS)in Guangdong, Peopleâs Republic of China, in February.Lancet. 362(9393), 1353â1358(2003)

2. Zhou, Y.C., Ma, Z.E., Brauer, F.:A discrete epidemic model for SARS transmission andcontrol in China .Mathematical Computer Modelling. 40(13),1491â1506 (2004)

3. Wang, W.D., Ruan, S.G.:Simulating the SARS outbreak in Beijing with limited data .Journal of Theoretical Biology. 227(3), 369â379 (2004)

4. Ellerin, T.:The new coronavirus: What we do- and donâtknow, Harvard Health Blog.(2020)

5. WHO novel coronavirus (2019-ncov) situation reports .DOI::https://www.who.int/e-mergencies/diseases/novelcoronavirus-2019/situation-reports.(2019)

6. Gralinski, L.E., Menachery, V.D.:Return of the coronavirus:2019-nCoV. Viruses.2020, 135., 135,(2020)

7. Furuya-Kanamori, L., Cox, M., Milinovich, G.J., Magalhaes, R.J.S., Mackay, I.M., Yakob,L.: Heterogeneous and dynamic prevalence of asymptomatic influenza virus infections.Emerging infectious diseases. 22, 1052-1056 (2016)

8. Heffernan, R.T., Pambo, B., Hatchett, R.J., Leman, P.A.,Swanepoel, R., Ryder, R.W.:Low seroprevalence of Ig $\mathrm{G}$ antibodies to Ebola virus in an epidemic zone:Ogooue-Ivindo region.Journal of Infectious Diseases. 191, 2005, (2005)

9. Dean, N.E., Halloran, M.E., Yang, Y., Longini, I.M.: Transmissibility and pathogenicity ofebola virus:a systematic review and meta-analysis of household secondary attack rate and asymptomatic infection.Clinical Infectious Diseases. 62, 1277 â1286 (2016)

10. Chua, L.O., Yang, L.: Cellular neural networks: theory. IEEE Trans. Circuits Syst[10]Robinson, M., Stilianakis, N.I.:A model for the emergence of drug resistance in the presence of asymptomatic infections .Mathematical Biosciences.243,163â177 (2013)

11. Grunnill, M.: An exploration of the role of asymptomatic infections in the epidemiology of engue viruses through susceptible, asymptomatic, infected and recovered (SAIR) models.Journal of Theoretical Biology.14, 195-204 (2018)

12. Robinson, M., Stilianakis, N.I.:A model for the emergence of drug resistance in the presence of asymptomatic infections. Mathematical Bioscie-nces. 243, 163-177(2013)

13. Tang, Y., Xiao, D., Zhang, W., Zhu, D.:Dynamics of epidemic models with asymptomatic Iifection and seasonal succession. Mathematical Biosciences and Engineering. 314, 1407 â1424 (2017)

14. Wu, S.S., Sun, P.P., Li, R.L., Zhao, L., Wang, Y.L., Jiang, L.F., Wang, H.L., and Deng, J. B.: Epidemiological Development of Novel Coronavirus Pneumonia in China .DOI:10.1101/2020.02.21.20026229.(2020)

15. Joseph, T.W., Kathy, L., and Gabriel, M.L.: Now casting and forecasting the potential domestic and international spread of the 2019-nCoV outbreak originating in Wuhan, China:a modelling study.Lancet. 395(10225), 689$697(2020)$
16. Liu, Y., Julian, W.T., and Tommy, T.Y.L.: Transmission dynamics of the COVID-19 epidemic in England.Int J Infect Dis. 104,132-138 (2021)

17. Yan Yue, Chen Yu, et al.:Modeling and prediction of New Coronavirus pneumonia epidemic based on a class of time-delay dynamic systems. Chinese Science: Mathematics. 50(03), 385-392 (2020)

18. Zhang Liying, Li Dongchen, Ren Jingli.:COVID-19 propagation analysis of multi-stage dynamic time-delay dynamic model.Journal of Wuhan University (Information Science Edition). 45(05), 658-666(2020)

19. Danane, Jaouad, Hammouch, Zakia, Singh, Jagdev, et al.:A fractional-order model of coronavirus disease 2019 (COVID-19) with governmental action and individual reaction.2021, DOI10.1002/mma.7759 (2021)

20. Furati, K. M.,Sarumi, I. O.,Khaliq, A. Q. M.:Fractional model for the spread of COVID-19 subject to government intervention and public perception.95,89-105 ( 2021)

21. Li Feng.:Dynamic modeling analysis and application of infectious diseases with asymptomatic infection[D].Zhongbei University,.DOI:10.27470/d.cnki.ghbgc.2021.000008.(2021)

22. Fei Yu, Zinan Zhang, Hui Shen, Yuanyuan Huang, Shuo Cai and Sichun Du.:FPGA implementation and image encryption application of a new PRNG based on a memristive Hopfield neural network with a special activation gradient.Chinese Physics B. DOI: 10.1088/16741056/ac3cb2(2021)

23. Fei Yu, Hui Shen, Zinan Zhang, et al.:Dynamics analysis, hardware implementation and engineering applications of novel multi-style attractors in a neural network under electromagnetic radiation. Chaos, Solitons Fractals.152, 111350 (2021)

24. Fei Yu, Hui Shen, Zinan Zhang, et al.:A new multiscroll Chua's circuit with composite hyperbolic tangentcubic nonlinearity: Complex dynamics, Hardware implementation and Image encryption application.Integration. 81, 71-83 (2021)

25. Hairong Lin, Chunhua Wang, Fei Yu, Cong Xu, Qinghui Hong, Wei Yao, Yichuang Sun.:An Extremely Simple Multiwing Chaotic System: Dynamics Analysis, Encryption Application, and Hardware Implementation.IEEE Transactions on Industrial Electronics. 68(12), 12708-12719 (2021)

26. Hairong Lin, Chunhua Wang, Chengjie Chen, Yichuang Sun, Chao Zhou, Cong Xu, Qinghui Hong.:Neural bursting and synchronization emulated by neural networks and circuits.IEEE Transactions on Circuits and Systems I.Regular Papers68(8),3397-3410 (2021)

27. Hairong Lin, Chunhua Wang, Quanli Deng, Cong Xu, Zekun Deng, Chao Zhou.: Review on chaotic dynamics of memristive neuron and neural network Nonlinear Dynamics.106 (1), 959-973(2021)

28. Xie Wenli, Wang Chunhua, Lin Hairong.:A fractionalorder multistable locally active memristor and its chaotic system with transient transition, state jump.Nonlinear Dynamics.104, 4523â4541 (2021)

29. Dreessche, P., Watmough, J.:Reproduction numbers and sub-threshold endemic equilibria for com-partmental models of disease transmission.Mathematical Biosciences. 180(1.2),29-48 (2002)

30. Diekmann, O., Heesterbeek, J., Metz.J.:On the definition and the computation of the basic reproduction ratio R0 in models for infectious diseases in heterogeneous populations.Journal of Mathematical Biology.28(4), 365â382 (1990) 
31. Diekmann,O.,Heesterbeek,J.,Roberts.:The construction of next generation matrices for compartmental epidemic models.Journal of the Royal Society Interface.7(47),873â885 (2010)

32. YanLi, YangQuan Chen,Igor Podlubny,cMittagâ Leffler stability of fractional order nonlinear dynamic systems.45(8),1965-1969 (2009)

33. E.Ahmed,A.M.A.El-Sayed,H.A.A.El-Saka,: $\quad$ Equilibrium points, stability and numerical solutions of fractional-order predator prey and rabies models, J.Math.Anal.Appl.325(1), 542 â553 (2007)

34. D.Matignon: Stability results for fractional differential equations with applications to control processing.Computational Engineering in Systems Applicationspp,963â968 (1996)

35. Diethelm K , Freed A D . :The FracPECE Subroutine for the Numerical Solution of Differential Equations of Fractional Order// Orschung Und Wissenschaftliches Rechnen: Beitrge Zum Heinz-billing-preis. (2002).

36. Garrappa R.:On linear stability of predictor-corrector algorithms for fractional differential equations. International Journal of Computer Mathematics.87(10-12):22812290 (2010). 